\title{
Short Instructions for Raman Microscope Horiba Xplora
}

\author{
Dejan Lazić
}

When laser light hits the sample most of the light is reflected with no shift in the frequency. Part of photones transfer energy to the sample which irradiates it with the shifted frequency. Fluorescence occurs when the sample absorbes energy and releases it slowely. Instantaneous changes in frequency are Raman scattering. Shift in frequency, Raman effect correlates to the energy difference between ground and virtual state. Emmited light of lower energy than the energy of laser are called Stokes lines and ones of higher energy are called anti-Stokes lines. They are formed if electron was excited before light hit it. Concidering the fact that more molecules are in the ground state, it is expected to see more Stokes than anti Stokes lines. Antistokes lines could be interesting if Stokes lines are masked with fluoresence.

\section{How to cite this book chapter:}

Lazić, D. 2019. Short Instructions for Raman Microscope Horiba Xplora. In: Vucelić Radović, B., Lazić, D. and Nikšić, M. (eds.) Application of Molecular Methods and Raman Microscopy/Spectroscopy in Agricultural Sciences and Food Technology, Pp. 199-206. London: Ubiquity Press. DOI: https://doi.org/10.5334/bbj.q. License: CC-BY 4.0 


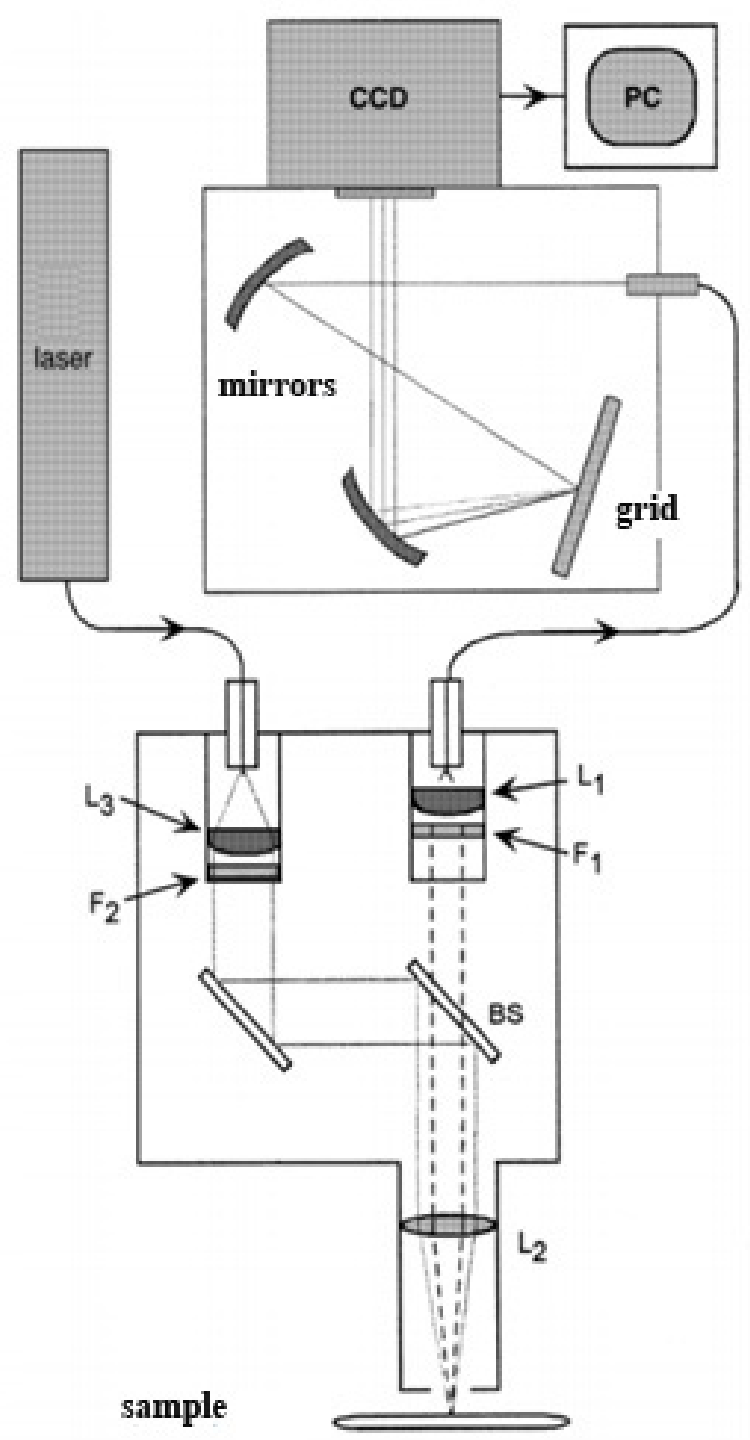

Figure 1: Construction of Raman microscope.

As Horiba Xplora instrument is the only Raman microscope at the Faculty of Agriculture further short instructions are aimed to facilitate its use by newly trained staff and $\mathrm{PhD}$ students in their reseach.

Turn on by pressing a switch on the extension cord. Switch on the computer, start LabSpec 6, icon on the middle of the screen.

Chech if lasers are powered on. 

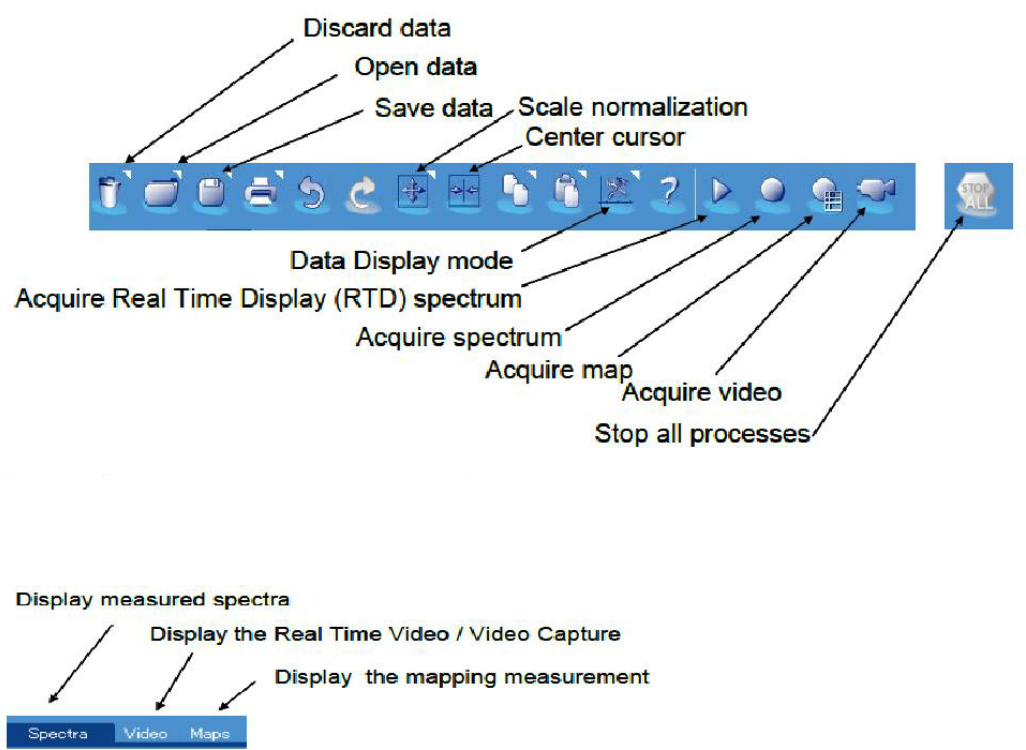

\section{Control panel}

The Control Panel on the right hand side includes all functionality required in LabSpec 6 to acquire, process, analyze and display data. The individual tabs of the Control Panel are organized as follows:

Analysis Display Methods Maintenance
Browser Acquisition Info Processing

Browser: view a list of all data opened in LabSpec6.

Acquisition: set up all acquisition parameters including hardware settings.

Info: view the information shell for each data file

Processing: data processing functions to modify raw data (including smoothing, baseline correction and math functions)

Analysis: data analysis functions to obtain information from the data Display: configure the display of spectra / video / mapping window

Methods: create customized multi-step one click sequences, data acquisition and analysis.

Maintenance: system calibration, Auto Calibration and Auto Alignment

\section{Autocalibration}

If you see a red $A C$ at the bothom of the screen autocalibration is required.

Figure 2: Basic commands on LabSpec 6. 


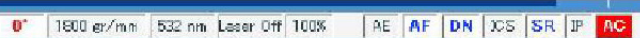

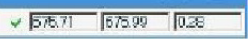

Place reference silica plate with marked lends $100 \mathrm{X}$ Sharpen

Press $\mathrm{AC}$, activates process of autocalibration

\section{Video acquisition}

Set filter weel on position 2

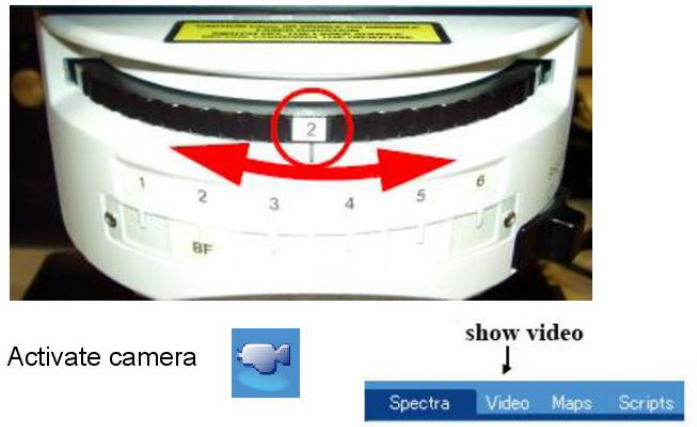

Set ojective in use

$$
\text { Objective } \quad \times 100
$$

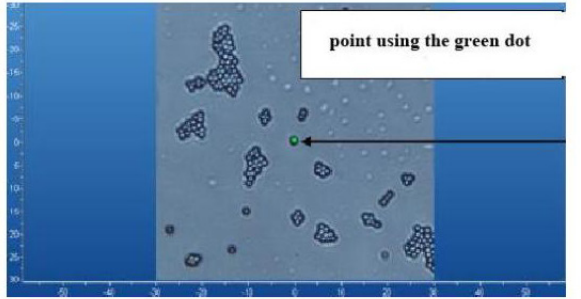

Stop,

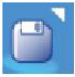

\section{Real Time Display}

Set filter weel on position 1

Figure 3: Video acquisition. 


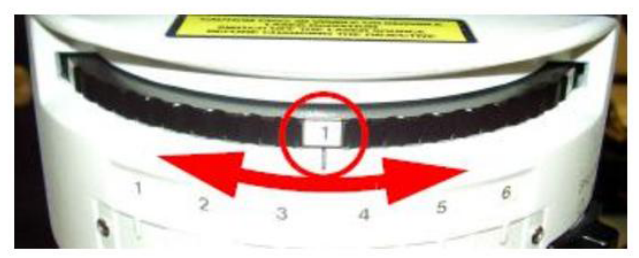

Set the spectrometer

\begin{tabular}{|c|c|c|}
\hline Objective & $\times 100$ & $\boldsymbol{\sigma}$ \\
\hline Grating & $1800 \mathrm{gr} / \mathrm{mm}$ & $\nabla$ \\
\hline ND Filter & $100 \%$ & $\nabla$ \\
\hline Laser & $633 \mathrm{~nm}$ & $\boldsymbol{\gamma}$ \\
\hline$\underline{\text { Slit }}$ & 150 & $b$ \\
\hline Hole & 70 & 1 \\
\hline
\end{tabular}

Set the RTD time

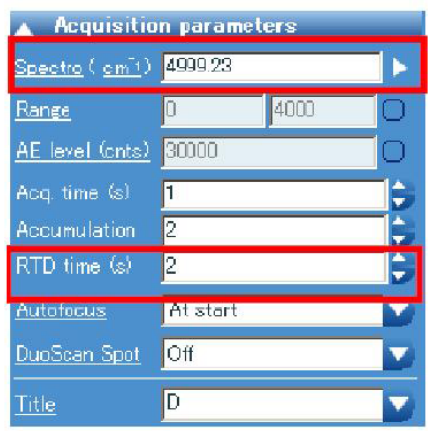

Greater number of lines gives better resolution but a narrower spectrum

Set laser intensity.

Set laser wavelenght.

Less gives beter resolution but lower intensity Smaler hole, better rezolution, lower intensity

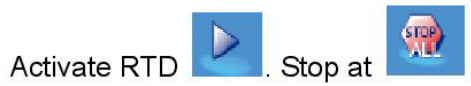

\section{Spectrum acquisition}

Set the filter weel to 1

Figure 4: Acquisition parameters. 


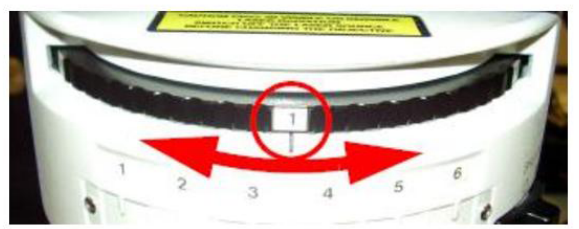

activate extended spectral range

imput necessery spectral range

Setacquisition time

Setthe accumulation
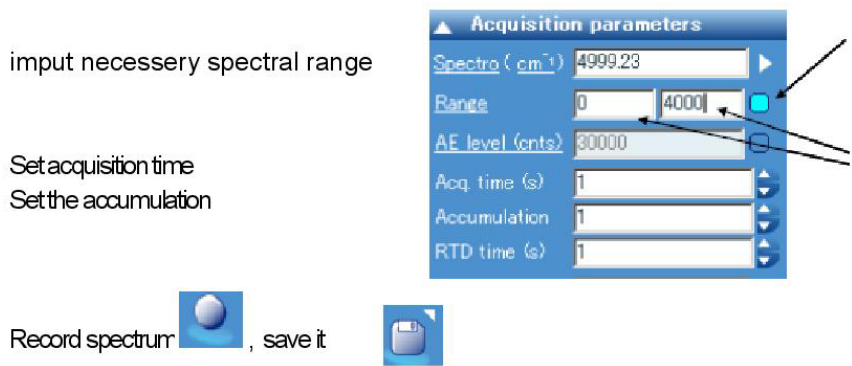

\section{Display mode of the spectra}

Recorded spectra are displayed in the Spectra data tab.

The display mode interface 2.. allows a user to change the Display mode of the spectra tab.

In order to zoom in to a specific spectral region, click in the graphical manipulation tool $Q$ bar and drag the target region of the spectrum with the cursor.

In order to rescale the spectral view (i.e., remove the zoom), click in the Icon in task bar, or right click on the spectrum and select "Rescale"

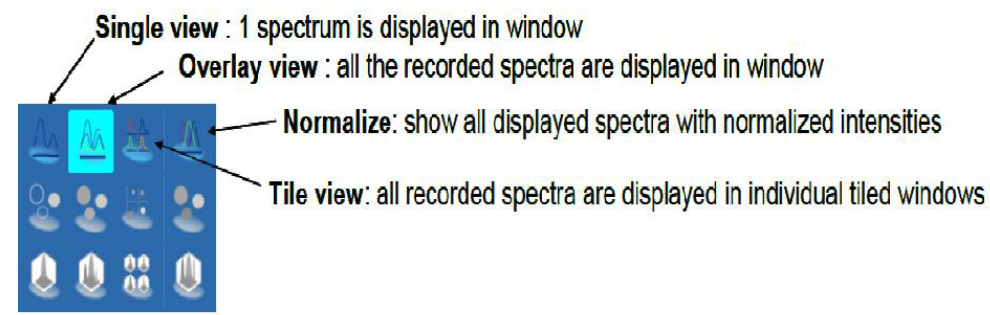

Figure 5: Options for viewing the spectrum. 


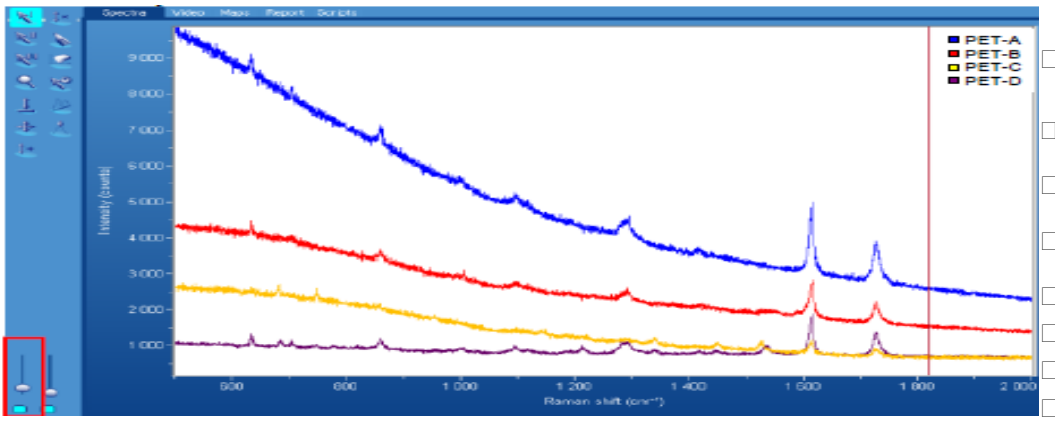

Figure 6: Overlay view of Raman spectra. 
